\section{Mood symptoms Associated with CADASIL Syndrome: A Case Report}

Asad Shaikh, MD and Joel Idowu, MD

Resident

\begin{abstract}
Objective. To discuss the psychiatric symptoms that are associated with CADASIL syndrome Abstract Cerebral:

Autosomal Dominant Arteriopathy with Subcortical Infarcts and Leukoencephalopathy (CADASIL) is a rare type of hereditary disease involving the small cerebral vessels. The clinical symptoms are various and include recurrent ischemic strokes, migraine with aura, seizures with epilepsy, psychiatric problems such as mood disturbances, and progressive cognitive decline leading to dementia. This disease needs awareness amongst the psychiatrists even though it is discussed much more in neurology literature. Psychiatric symptoms are seen in $20-41 \%$ of patients with CADASIL syndrome $(1,2)$. Psychiatric symptoms are actually the initial presentation in $15 \%$ of the cases. (3) The psychiatric disturbance most reported are mood disturbances (9-41\%) especially depression. Here a 42-year-old African American female was brought to the hospital emergency room after she was found wandering in the streets. Psychiatry was consulted for altered mental status. Upon evaluation by the psychiatric consult service she was only oriented to person, depressed, anxious and complaining of headaches. Initial CT scan showed marked small vessel disease and old lacunar infarcts in the basal ganglia and right corona radiata. Magnetic Resonance Imaging (MRI) of the brain showed acute infarcts in the right posterior frontal and right parietal lobes along with old infarcts. Her symptoms and findings on imaging were consistent with CADASIL syndrome. Once the diagnosis was confirmed and prior records were obtained patient was resumed on an antidepressant and anxiolytic.

Conclusion. The purpose of this case report was to discuss psychiatric symptoms associated with CADASIL syndrome. Although there has been research showing a relationship between vascular disease and depression, a review of the literature suggests that there needs to be more research done to explore other psychiatric disturbances that may be seen with this syndrome. Psychiatric symptoms that are untreated can have the potential to further impact the quality of life therefore psychiatrists need to be aware of this syndrome in order to treat these patients promptly. References. $1 \mathrm{https} / / \mathrm{bmcmedicine.biomedcentral.com/arti-}$ cles/10.1186/s12916-017-0778-8 2 http://dx.doi.org/10.32474/ OJNBD.2018.01.000101 3 https://pdfs.semanticscholar.org/47f6/5952ee3c5dcf2a61345f704914b17fa8dc0d.pdf
\end{abstract}

\section{Psychiatric Prescriber Attitudes, Experiences, and Proclivities Toward Digital Medicine and How They Influence Adoption of Digital Medicine Platforms}

Charles Ruetsch, $\mathrm{PhD}^{1}$, Dawn Velligan, $\mathrm{PhD}^{2}$, Delbert Robinson, $\mathrm{MD}^{3}$, Chris Jaeger, MD, MBA ${ }^{4}$, William Carpenter, $\mathrm{MD}^{5}$, Tigwa Davis, $\mathrm{PhD}^{1}$, Joshua N. Liberman, $\mathrm{PhD}^{1}$, Jennifer Clerie, $\mathrm{BS}^{1}$, Heidi Waters, MBA, $\mathrm{PhD}^{6}$ and Felicia Forma, $\mathrm{BSc}^{6}$

${ }^{1}$ Health Analytics, LLC, Columbia, MD, USA, ${ }^{2}$ University of Texas Health Science Center, San Antonio, TX, USA, ${ }^{3}$ The Donald and Barbara Zucker School of Medicine at Hofstra/Northwell, Glen Oaks, NY, USA, ${ }^{4} \mathrm{JHC}$ Solutions, LLC, San Francisco, CA, USA, ${ }^{5}$ Maryland Psychiatric Research Center, University of Maryland School of Medicine, Baltimore, MD, USA, and ${ }^{6}$ Otsuka Pharmaceutical Development \& Commercialization, Inc., Princeton, NJ, USA

Presenting Author: Charles Ruetsch

\begin{abstract}
Background. Psychiatric prescribers typically assess adherence by patient or caregiver self-report. A new digital medicine (DM) technology provides objective data on adherence by using an ingestible event monitoring (IEM) sensor embedded within oral medication to track ingestion. Despite likely clinical benefit, adoption by prescribers will in part depend on attitudes toward and experience with digital health technology, learning style preference (LSP), and how the technology s utility and value are described.

Objective. is to identify attitudes, experiences, and proclivities toward DM platforms that may affect adoption of the IEM platform and provide direction on tailoring educational materials to maximize adoption. Methods A survey of prescribers treating seriously mentally ill patients was conducted to assess drivers/ barriers to IEM adoption. Factor analysis was performed on 13 items representing prior experience with and attitudes toward DM. Factor scores were correlated with prescriber characteristics including attitude and experience with digital technologies, LSP, and level of focus on healthcare cost.

Results. A total of 127 prescribers (56\% female, $76 \%$ physicians, mean age 48.1 yrs.) completed the survey. Over $90 \%$ agreed medication adherence is important, visits allow enough time to monitor adherence $(84.1 \%)$, and tailoring treatment to level of adherence would be beneficial (92.9\%). The majority (65.9\%) preferred relying upon outcomes data as their learning style while $15.9 \%$ preferred opinion leader recommendations and $18.3 \%$ information about how the technology would affect practice efficiency. Factor
\end{abstract}

